\title{
Smart Sensor Platforms and Systems for Aerospace Applications
}

\author{
Gary W. Hunter ${ }^{1}$, Darby B. Makel ${ }^{2}$, Jennifer C. Xu ${ }^{1}$ \\ ${ }^{1}$ NASA Glenn Research Center, 21000 Brookpark Rd., Cleveland, OH, USA \\ ${ }^{2}$ Makel Engineering, Inc, Chico, CA, USA. \\ Gary.W.Hunter@nasa.gov
}

\begin{abstract}
Aerospace applications require a variety of sensing technologies to monitor conditions related to both space exploration and aeronautic aircraft operations. These sensing technologies have requirements beyond those of standard commercial applications often due to the flight conditions and harsh environments involved. This paper is a broad overview of efforts to produce Smart Sensor Platforms and Systems that can be tailored to measure multiple chemical species for aerospace applications. A Smart Sensor System as described here implies the use of sensors combined with electronic processing capability and other supporting technologies. Applications include leak detection, engine emissions monitoring, planetary exploration, fire detection, human health monitoring, and environmental monitoring. These efforts range from development of base sensor platforms to the evaluation of more mature systems in relevant environments. Examples will be given of microsensor platform technology, smart sensor systems, and harsh environment sensor systems. Although Smart Sensor Systems can have a significant impact on aerospace applications, extensive relevant demonstration testing is necessary for their long-term implementation. It is suggested that small, smart sensor system technologies are an enabling first step towards more intelligent vehicle systems.
\end{abstract}

Key words: Smart Sensor, Microsensor, Wireless, Aerospace, Harsh Environment

\section{Introduction}

Aerospace applications require a variety of sensing technologies to monitor conditions related to both space exploration and aeronautic aircraft operations [1-3]. Improved sensing technology is necessary to increase safety, reduce emissions, improve performance, and in general enable more intelligent systems. Often the requirements for chemical sensor systems in aerospace applications are notably beyond that of commercial applications. These include not only minimal size, weight, and power consumption to avoid impact to the vehicle system, but also a wide range of possible operational requirements including: functionality in environments ranging from cryogenic conditions to high temperature; detection of species at ppm concentration or below to exposure to pure gases; and detection of multiple species simultaneously down to accurate detection of a single species of interest.

The approach described in this paper to address aerospace chemical sensing applications needs has been to develop core technologies, esp. smart platforms and systems, for a range of applications. The approach is to drive intelligence to a local level to enable a long-term vision of intelligent aerospace system [4-5].

This paper provides a broad overview of this approach related to chemical sensor systems including: Microsensor Platforms; Integration of these Platforms into complete Smart Sensor Systems; and an example Harsh Environment Aerospace Application. It is suggested that small, smart sensor system technologies are an enabling first step in acquiring information about an aerospace system or environment, leading to more intelligent systems with cognition and decision-making capabilities.

\section{Microsensor Platforms}

A range of chemical microsensors are being developed to meet of aerospace applications based in part on microfabrication technology producing sensors with minimal size, weight, and power consumption. In order to meet the broad needs of aerospace applications, core microsensor platforms have been developed. The major platforms used are Schottky diodes (Figure 1), resistance-based sensors, and electrochemical cells. Modifications of these sensors platforms related to materials used, operating conditions, and packaging have enabled the capability to address a wide range of applications. Using this approach, sensors to 
detect species including hydrogen, hydrocarbons, nitrogen oxides, carbon monoxide, carbon dioxide, and oxygen have been developed. The minimal size, weight, and power consumption of the microsensors allow their integration into hardware to form Smart Sensor Systems.

Figure 1. Examples of a microsensor platform: Schottky Diode and Resistive Based Sensor [2]

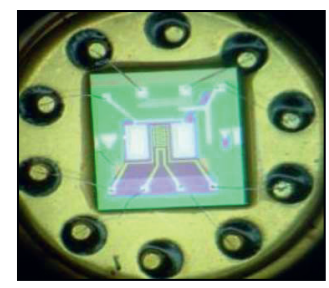

\section{Smart Sensor Systems}

A Smart Sensor System is, at a minimum, the combination of a sensing element with processing capabilities provided by a microprocessor. A more expansive view of a Smart Sensor System is a complete selfcontained sensor system that includes the capabilities for data logging and processing, self-contained power, and an ability to transmit or display informative data to an outside user [6]. Figure 2 shows an example Smart Sensor System for leak detection. This system includes a sensor array to determine the concentration of hydrogen, hydrocarbons, and oxygen, a microprocessor, wired or wireless communication, and integrated or vehicle power [2]. This core smart sensors hardware has been used across a range of aerospace applications for vastly different operational environments [6], and the approach continues to expand to meet the needs of new applications.

Figure 2.

Prototype version of a smart leak sensor system with supporting electronics including signal conditioning and telemetry [2].

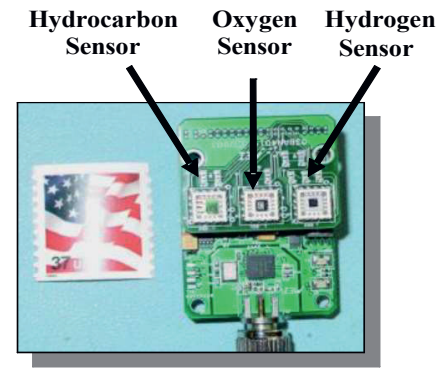

\section{Harsh Environment Applications}

One aerospace challenge for the Smart Sensor System approach is application in extreme environments where both the core sensor technology and supporting hardware approach their operational limits. For examples, engine emissions monitoring includes measurement of the chemical species from the exit of jet engines. Specific packaging has been developed to enable operation of the sensors in such harsh environments and detection of changes in engine operational state has been shown [7]. For potential engine applications, such an emissions monitoring system could use conventional electronics mounted in benign locations in the vehicle. However, although Venus planetary applications could use the same core sensor microplatforms [8], extended operation on the Venus surface is not viable with conventional electronics and thus new high temperature electronics are being developed for this application with a simpler operating approach. Status of this Venus application will be discussed highlighting both the challenges and possibilities of such technology.

\section{References}

[1] G. W. Hunter, J. C. Xu, and D. B. Makel, Case Studies in Chemical Sensor Development, BioNanoFluidic MEMS, Edited by Hesketh, P.J., Springer US. Chapter 8, 2008

[2] G. W. Hunter, J. C. Xu, P. G. Neudeck,,D. B. Makel B. Ward, and C. C. Liu, "Intelligent Chemical Sensor Systems For In-Space Safety Applications', 42nd AIAA/ASME/SAE/ASEE Joint Propulsion Conference And Exhibit, Sacramento, CA, 9-12 July 2006, Paper AIAA-2006-4356

[3] G. W. Hunter, J. C. Xu, A. M. Biaggi-Labiosa, B. Ward, P. Dutta, and C.C. Liu, Smart Sensor Systems for Spacecraft Fire Detection and Air Quality Monitoring, in 40th International Conference On Environmental Systems, AIAA: Portland, Oregon, 2011, Vol. AIAA 2011-5021

[4] G. W. Hunter and A. Behbahani, A Brief Review of the Need for Robust Smart Wireless Sensor Systems for Future Propulsion Systems, Distributed Engine Controls, and Propulsion Health Management, 58th International Instrumentation Symposium, San Diego, CA, June 4-78, 2012

[5] G. Hunter, J. Xu, L. Evans, A. Biaggi-Labiosa, B. Ward, S. Rowe, D.Makel, C.C. Liu, P. Dutta, G. Berger and R. VanderWal, Micro/nanoscale chemical- sensor systems for aerospace applications, June 2010, SPIE Newsroom. DOI: $10.1117 / 2.1201006 .002984$

[6] G. W. Hunter, J. R. Stetter, P. J. Hesketh, and C.C. Liu, Smart Sensor Systems, Interface Magazine, Electrochemical Society Inc., Vol. 20, no. 1, Winter, 66-69, 2011

[7] G. W. Hunter; D. L. Simon; J. C. Xu; A. BiaggiLabiosa; S. Carranza; D. Makel, Aircraft Ground Demonstration of Engine Emissions Monitoring System Based on a Gas Microsensor Array, AIAA-2014-3925, 50th AIAA/ASME/ SAE/ASEE Joint Propulsion Conference, July 2014, Cleveland, $\mathrm{OH}$

[8] Darby Makel, Jennifer Xu, Gary Hunter, Harsh Environment Gas Sensor Array for Venus Atmospheric Measurements, Venus Science Priorities for Laboratory Measurements in Instrumentation Definition Workshop, April, 2015, Hampton, VA 